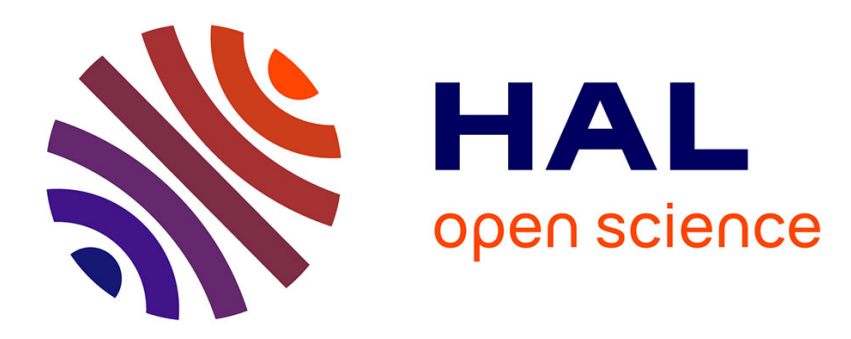

\title{
Le modèle stochastique SIS pour une épidémie dans un environnement aléatoire
}

\author{
Nicolas Bacaër
}

\section{To cite this version:}

Nicolas Bacaër. Le modèle stochastique SIS pour une épidémie dans un environnement aléatoire. Journal of Mathematical Biology, 2016, 73 (4), pp.847 - 866. 10.1007/s00285-016-0974-8 . hal$01414037 \mathrm{v} 4$

\section{HAL Id: hal-01414037 \\ https://hal.science/hal-01414037v4}

Submitted on 20 Jun 2020

HAL is a multi-disciplinary open access archive for the deposit and dissemination of scientific research documents, whether they are published or not. The documents may come from teaching and research institutions in France or abroad, or from public or private research centers.
L'archive ouverte pluridisciplinaire HAL, est destinée au dépôt et à la diffusion de documents scientifiques de niveau recherche, publiés ou non, émanant des établissements d'enseignement et de recherche français ou étrangers, des laboratoires publics ou privés. 


\title{
Le modèle stochastique SIS pour une épidémie dans un environnement aléatoire
}

\author{
Nicolas Bacaër* † \\ J. Math. Biol. 73 (2016) 847-866. \\ doi : 10.1007/s00285-016-0974-8 \\ hal : 01414037 \\ Traductions : [ar, de, es, it, ja, nl, pt, ru, zh], html]
}

\begin{abstract}
Résumé
Dans un environnement aléatoire qui est une chaîne de Markov en temps continu à deux états, le temps moyen jusqu'à extinction du modèle stochastique SIS pour une épidémie croît, dans le cas surcritique, exponentiellement par rapport à la taille de la population si les deux états sont favorables, et suivant une loi de puissance si l'un des états est favorable alors que l'autre est défavorable à la propagation.
\end{abstract}

\section{Introduction}

Soit $N$ la taille de la population, supposée constante. Dans le modèle stochastique SIS pour une épidémie, chaque individu est soit susceptible (S) d'attraper la maladie, soit infecté (I) (Nåsell, 2011). Imaginons que l'environnement suive une chaîne de Markov en temps continu à deux états, $q_{j}(j=1,2)$ étant le taux de sortie de l'état $j$. Dans l'environnement $j$, le taux de contact de chaque individu est $a_{j}>0$. Si à un instant $t$ le nombre de personnes infectées est $n$ et l'environnement est $j$, la probabilité d'avoir une nouvelle infection entre $t$ et $t+d t$, avec $d t$ infiniment petit, est $a_{j} n(1-n / N) d t$. Supposons que le taux de guérison $b>0$ d'un individu soit le même dans les deux environnements : la probabilité d'une nouvelle guérison dans la population est alors $b n d t$ entre $t$ et $t+d t$. Ce modèle a été étudié par Artalejo et al. (2013) mais pas dans la limite où la taille $N$ de la population devient grande. C'est d'ailleurs un cas particulier de processus de naissance et de mort dans un environnement markovien (Cogburn et Torrez, 1981).

\footnotetext{
*Institut de Recherche pour le Développement, Bondy, France. Courriel : nicolas.bacaer@ird.fr

${ }^{\dagger}$ Université Pierre et Marie Curie, Les Cordeliers, Paris, France.
} 


$$
\begin{aligned}
& \text { Posons } \\
& Q=\left(\begin{array}{cc}
-q_{1} & q_{2} \\
q_{1} & -q_{2}
\end{array}\right), \quad u_{1}=\frac{q_{2}}{q_{1}+q_{2}}, \quad u_{2}=\frac{q_{1}}{q_{1}+q_{2}}, \quad R_{0}=\frac{a_{1} u_{1}+a_{2} u_{2}}{b} .
\end{aligned}
$$

Alors $Q$ est le générateur infinitésimal, tandis que $u_{1}$ (resp. $u_{2}$ ) est la proportion du temps passé dans l'environnement 1 (resp. 2). Pour le modèle linéarisé, à savoir le processus linéaire de naissance et de mort dans un environnement aléatoire de paramètres $a_{j}$ et $b$, Bacaër et Ed-Darraz (2014) notent qu'il y a extinction presque sûrement si et seulement si $R_{0} \leq 1$. Pour le modèle nonlinéaire SIS en revanche, il y a extinction quelle que soit la valeur du paramètre $R_{0}$. Cependant, lorsque la population $N$ est grande, le temps moyen jusqu'à extinction est grand quand $R_{0}>1$ et petit quand $R_{0}<1$. Soit $T_{n, j}$ ce temps partant de $n$ personnes infectées $(0 \leq n \leq N)$ dans l'environnement $j(j=1,2)$. Alors pour tout $1 \leq n \leq N$,

$$
\begin{aligned}
& T_{n, 1}=\frac{1+a_{1} n(1-n / N) T_{n+1,1}+b n T_{n-1,1}+q_{1} T_{n, 2}}{a_{1} n(1-n / N)+b n+q_{1}}, \\
& T_{n, 2}=\frac{1+a_{2} n(1-n / N) T_{n+1,2}+b n T_{n-1,2}+q_{2} T_{n, 1}}{a_{2} n(1-n / N)+b n+q_{2}} .
\end{aligned}
$$

De plus $T_{0,1}=T_{0,2}=0$. L'objectif de cet article est d'étudier dans le cas surcritique, c'est-à-dire quand $R_{0}>1$, le comportement du temps moyen jusqu'à extinction lorsque la taille de la population $N$ devient grande.

Dans un environnement constant avec un taux de contact $a$ tel que $R_{0}=$ $\frac{a}{b}>1$, Doering et al. (2005, Tableau 1) montrent que le temps moyen jusqu'à extinction partant d'une fraction infectée de la population $x=n / N$ croît quand $N \rightarrow \infty$, indépendamment de $x$, comme $\frac{a}{(a-b)^{2}} \sqrt{\frac{2 \pi}{N}} e^{c N}$, où $c=b / a-1-$ $\log (b / a)$. Autrement dit, le temps moyen croît à peu près exponentiellement avec $N$. Bacaër (2015) suggère un résultat similaire lorsque l'environnement varie de manière périodique, même s'il n'y a pas de formule explicite pour le paramètre de croissance $c$.

L'article qui suit suggère que dans un environnement aléatoire surcritique, c'est-à-dire avec $R_{0}>1$, deux régimes doivent être distingués : un régime fortement surcritique où $a_{1}>a_{2}>b$ et un régime faiblement surcritique où $a_{1}>b>a_{2}$. Dans le cas fortement surcritique où $a_{1}>a_{2}>b$ (ce qui implique d'ailleurs que $R_{0}>1$ ), on conjecture que le temps moyen jusqu'à extinction croît à peu près exponentiellement avec $N$ avec un paramètre $c$ égal à $c_{2}=b / a_{2}-1-\log \left(b / a_{2}\right)$. Plus exactement, le logarithme de ce temps moyen, divisé par $N$, tend vers $c_{2}$. Le comportement asymptotique est donc le même que si l'environnement restait bloqué dans l'état 2, c'est-à-dire celui le moins favorable à la propagation de l'épidémie et celui dans lequel l'extinction a lieu préférentiellement.

En revanche, si $a_{1}>b>a_{2}$ et $R_{0}>1$, alors on conjecture que le temps moyen jusqu'à extinction croît comme $N^{\omega}$ avec

$$
\omega=\frac{q_{2}}{b-a_{2}}-\frac{q_{1}}{a_{1}-b} .
$$


Dans ce cas, $R_{0}>1$ équivaut à $\omega>0$. De manière intuitive, il arrive de temps en temps que l'on reste dans l'état 2 pendant une durée très longue. La probabilité que cette durée soit supérieure à $\tau$ est $e^{-q_{2} \tau}$. Dans l'état 2, le temps moyen d'extinction pour $N$ grand est de l'ordre de $\tau \simeq \frac{\log N}{b-a_{2}}$ (Doering et al., 2005) ; cela se devine aussi avec l'équation de quasi-extinction $e^{-\left(b-a_{2}\right) \tau}=1 / N$. Donc finalement la probabilité d'extinction lors d'une période dans l'état 2 est de l'ordre de $e^{-q_{2} \tau} \simeq N^{-\frac{q_{2}}{b-a_{2}}}$. Ceci suggère que le temps moyen jusqu'à extinction est de l'ordre de grandeur de l'inverse, c'est-à-dire $N^{\frac{q_{2}}{b-a_{2}}}$. Selon notre analyse, l'ordre de grandeur est en réalité $N^{\omega}$ avec $\omega$ donné par (3).

Notons d'ailleurs que si $q_{1}$ et $q_{2}$ sont multipliés par $1+\varepsilon$ avec $\varepsilon>0$, alors $R_{0}$ reste inchangé mais $\omega$ est multiplié par $1+\varepsilon$ : dans ce modèle, une variabilité environnementale accrue tend à augmenter le temps moyen d'extinction.

Cette loi de puissance en $N^{\omega}$ semble pouvoir se généraliser au cas d'un environnement suivant une chaîne de Markov irréductible à un nombre d'états $J$ quelconque avec $a_{1}>a_{2}>\ldots>a_{J}$, pourvu que $b>a_{J}$ et $R_{0}>1$. Avec un générateur infinitésimal $Q$, on a $R_{0}=\left(\sum_{j=1}^{J} a_{j} u_{j}\right) / b$, $u$ étant l'unique vecteur tel que $Q u=0$ et $\sum_{j=1}^{J} u_{j}=1$ (Bacaër et Ed-Darraz, 2014). Notons $A$ et $B$ les matrices diagonales $\operatorname{diag}\left(a_{1}, \ldots, a_{J}\right)$ et $\operatorname{diag}(b, \ldots, b)$. Considérons la borne spectrale $\mu(s)=\max \{\Re(\lambda) ; \lambda \in \sigma(Q-s(A-B))\}$, où $\sigma(\cdot)$ désigne le spectre d'une matrice et $\Re(\cdot)$ la partie réelle d'un nombre complexe. On montre qu'il existe un unique $\omega>0$ tel que $\mu(\omega)=0$. On conjecture alors que le temps moyen jusqu'à extinction croît comme $N^{\omega}$. Dans le cas particulier où $a_{j} \neq b$ pour tout $j$, le nombre $\omega$ est simplement la valeur propre de plus grande partie réelle de la matrice $(A-B)^{-1} Q$. Cette matrice et l'expression de sa valeur propre $\omega$ donnée par (3) pour $I=2$ se trouvent déjà chez Sawyer et Slatkin (1981, Eq. (14) et (20)) pour un modèle de population sans stochasticité démographique dans le cas sous-critique $\left(R_{0}<1\right)$; dans ce cas, le temps moyen jusqu'à extinction croît cependant comme le logarithme de $N$.

Plusieurs auteurs ont trouvé une loi de puissance pour le temps moyen jusqu'à extinction dans des modèles de population en environnement aléatoire mais avec un bruit blanc au lieu d'une chaîne de Markov (Ludwig, 1976; Leigh, 1981; Lande, 1993). Ils utilisent comme point de départ une équation de diffusion. Cependant Gaveau et al. (1996) et Doering et al. (2005) ont montré que cette équation conduit à une formule inexacte pour le paramètre $c$ même dans un environnement constant. En effet, cette équation ne représente pas toujours bien la queue de la distribution quasi-stationnaire, qui est précisément la partie la plus importante pour étudier le processus d'extinction. On notera cependant que Kamenev et al. (2008) ont abordé le problème de l'extinction pour des populations dans un environnement aléatoire avec un bruit coloré en utilisant des intégrales de chemin.

La section 2 présente la distribution quasi-stationnaire du modèle. La section 3 utilise la méthode de Brillouin, Kramers et Wentzel (BKW) pour obtenir des approximations valables dans les zones du paramètre $x=n / N$ où la distribution varie rapidement. La section 4 utilise une autre approximation dans la zone où la distribution varie lentement. La section 5 utilise une équation 
de diffusion pour raccorder les deux précédentes approximations : les fonctions hypergéométriques confluentes de Kummer et Tricomi remplacent ici la fonction d'erreur de Gauss utilisée notamment par Meerson et Sasorov (2008). La section [6 estime la valeur propre associée avec la distribution quasi-stationnaire. Aucune de ces approximations n'ayant été obtenue de manière rigoureuse, la section 7 présente néanmoins des simulations numériques qui semblent confirmer la valeur numérique des exposants pour la loi exponentielle dans le cas fortement surcritique et pour la loi de puissance dans le cas faiblement surcritique. La section 8 ajoute quelques remarques. En particulier, on compare dans le régime faiblement surcritique le cas d'un environnement markovien et celui d'un environnement périodique avec la même proportion du temps dans un état favorable ou défavorable : le premier conduit à une une loi de puissance pour le temps moyen jusqu'à extinction, le second à une loi exponentielle. La section 9 considère le cas d'une chaîne de Markov avec un nombre fini d'états et évoque d'autres généralisations. Enfin la section 10 se penche sur les environnements à la fois périodiques et stochastiques, mimant ainsi les saisons et la variabilité climatique.

\section{La distribution quasi-stationnaire}

Considérons la probabilité $p_{n, j}(t)$ d'avoir $n$ personnes infectées dans l'environnement $j$ au temps $t$. Alors

$$
\begin{aligned}
\frac{d p_{n, 1}}{d t}= & -\left[a_{1} n\left(1-\frac{n}{N}\right)+b n+q_{1}\right] p_{n, 1}+b(n+1) p_{n+1,1} \\
& +a_{1}(n-1)\left(1-\frac{n-1}{N}\right) p_{n-1,1}+q_{2} p_{n, 2} \\
\frac{d p_{n, 2}}{d t}= & -\left[a_{2} n\left(1-\frac{n}{N}\right)+b n+q_{2}\right] p_{n, 2}+b(n+1) p_{n+1,2} \\
& +a_{2}(n-1)\left(1-\frac{n-1}{N}\right) p_{n-1,2}+q_{1} p_{n, 1}
\end{aligned}
$$

Soit $P$ le vecteur colonne $\left(p_{0,1}, p_{0,2}, p_{1,1}, p_{1,2}, \ldots, p_{N, 1}, p_{N, 2}\right)$. Considérons la matrice diagonale d'ordre deux $A_{n}=\operatorname{diag}\left[a_{1} n\left(1-\frac{n}{N}\right), a_{2} n\left(1-\frac{n}{N}\right)\right]$. De même, posons $B_{n}=\operatorname{diag}[b n, b n]$. Posons par ailleurs

$$
M=\left(\begin{array}{ccccc}
Q & B_{1} & 0 & \cdots & 0 \\
0 & Q-A_{1}-B_{1} & B_{2} & \ddots & \vdots \\
0 & A_{1} & Q-A_{2}-B_{2} & \ddots & 0 \\
\vdots & \ddots & \ddots & \ddots & B_{N} \\
0 & \cdots & 0 & A_{N-1} & Q-B_{N}
\end{array}\right)=\left(\begin{array}{c|c}
Q & * \\
\hline 0 & R
\end{array}\right) .
$$

Le système (4)-(5) est de la forme $\frac{d P}{d t}=M P$. L'ensemble des valeurs propres de $M$ est la réunion de celles de $Q$ et de celles de $R$. Les valeurs propres de $Q$ sont 0 et $-\left(q_{1}+q_{2}\right)$. Les coefficients de la matrice $R$ en dehors de la diagonale sont positifs ou nuls. De plus la matrice $R$ est irréductible, comme on 
peut le voir avec le graphe associé. Un corollaire du théorème de Perron et Frobenius Smith, 1995, Corollaire 3.2) montre que la matrice $R$ a une valeur propre réelle $\lambda$ qui est simple, strictement supérieure à la partie réelle de toutes les autres valeurs propres, et avec un vecteur propre associé dont toutes les composantes sont strictement positives. Par ailleurs, en notant $\mathbf{1}$ le vecteur ligne $[1,1, \ldots, 1]$ de taille $2 N$, on voit que $1 R=[-b,-b, 0,0, \ldots, 0,0]$; d'après Berman et Plemmons (1994, Théorème 6.2.3, L33), ceci implique que $\lambda<0$. Soit $\pi=\left(\pi_{0,1}, \pi_{0,2}, \pi_{1,1}, \pi_{1,2}, \ldots, \pi_{N, 1}, \pi_{N, 2}\right)$ un vecteur propre de $M$ associé à la valeur propre $\lambda: M \pi=\lambda \pi$, autrement dit,

$$
\begin{aligned}
\lambda \pi_{n, 1}= & -\left[a_{1} n\left(1-\frac{n}{N}\right)+b n+q_{1}\right] \pi_{n, 1}+b(n+1) \pi_{n+1,1} \\
& +a_{1}(n-1)\left(1-\frac{n-1}{N}\right) \pi_{n-1,1}+q_{2} \pi_{n, 2} \\
\lambda \pi_{n, 2}= & -\left[a_{2} n\left(1-\frac{n}{N}\right)+b n+q_{2}\right] \pi_{n, 2}+b(n+1) \pi_{n+1,2} \\
& +a_{2}(n-1)\left(1-\frac{n-1}{N}\right) \pi_{n-1,2}+q_{1} \pi_{n, 1} .
\end{aligned}
$$

Normalisons le vecteur propre en imposant par exemple que $\frac{1}{N} \sum_{n=1}^{N}\left(\pi_{n, 1}+\right.$ $\left.\pi_{n, 2}\right)=1$. La figure 1 montre $\pi_{n, 1}$ et $\pi_{n, 2}$ en fonction de $n / N$ pour $n \geq 1$ dans deux cas : un cas où $R_{0}>1$ et $a_{1}>a_{2}>b$, un cas où $R_{0}>1$ et $a_{1}>b>a_{2}$. Le vecteur propre $\pi$ est calculé avec le logiciel Scilab. Le pic de $\pi_{n, 1}$ est proche de $x_{1}=1-b / a_{1}$, comme on pourrait s'y attendre en pensant à l'équation de champ moyen $\frac{d i}{d t}=a_{1} i(1-i)-b i$ pour la proportion $i$ de personnes infectées. En effet $x_{1}$ est le seul état d'équilibre stable de cette équation. Le pic de $\pi_{n, 2}$ est proche de $x_{2}=1-b / a_{2}$ si $a_{2}>b$ et proche de $x=0$ si $a_{2}<b$.
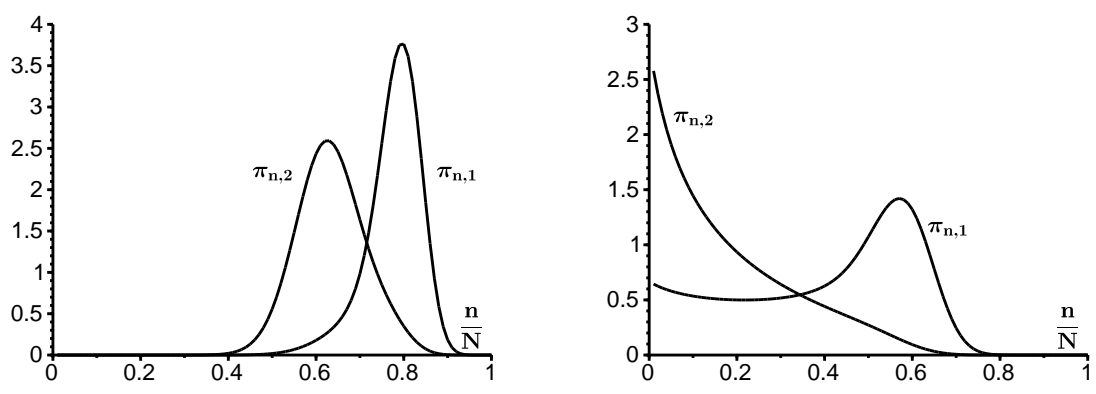

Figure 1 - Les composantes $\pi_{n, 1}$ et $\pi_{n, 2}$ pour $n \geq 1$ du vecteur propre $\pi$ en fonction de $n / N$ lorsque $N=100, q_{1}=1$ et $q_{2}=1$. Autres valeurs des paramètres : a) $a_{1}=10>a_{2}=5>b=2$; b) $a_{1}=5>b=2>a_{2}=1$.

Posons $\hat{\pi}=\left(\pi_{1,1}, \pi_{1,2}, \ldots, \pi_{N, 1}, \pi_{N, 2}\right)$ et $\hat{T}=\left(T_{1,1}, T_{1,2}, \ldots, T_{N, 1}, T_{N, 2}\right)$. Le système linéaire (11)-(2) peut s'écrire sous la forme

$$
\begin{aligned}
& -1=q_{1}\left(T_{n, 2}-T_{n, 1}\right)+a_{1} n\left(1-\frac{n}{N}\right)\left(T_{n+1,1}-T_{n, 1}\right)+b n\left(T_{n-1,1}-T_{n, 1}\right), \\
& -1=q_{2}\left(T_{n, 1}-T_{n, 2}\right)+a_{2} n\left(1-\frac{n}{N}\right)\left(T_{n+1,2}-T_{n, 2}\right)+b n\left(T_{n-1,2}-T_{n, 2}\right) .
\end{aligned}
$$


Les systèmes (8)-(9) et (6)-(7) montrent que $-\mathbf{1}^{*}=R^{*} \hat{T}$ et $\lambda \hat{\pi}=R \hat{\pi}$, où l'étoile * désigne la transposition de vecteurs ou de matrices. En prenant le produit scalaire usuel, on obtient $\left\langle-\mathbf{1}^{*}, \hat{\pi}\right\rangle=\left\langle R^{*} \hat{T}, \hat{\pi}\right\rangle=\langle\hat{T}, R \hat{\pi}\rangle=\lambda\langle\hat{T}, \hat{\pi}\rangle$. Donc

$$
-\frac{1}{\lambda}=\frac{\langle\hat{T}, \hat{\pi}\rangle}{\left\langle\mathbf{1}^{*}, \hat{\pi}\right\rangle} .
$$

Autrement dit, $-1 / \lambda$ est une combinaison linéaire des $\left(T_{n, j}\right)$ pour $n \geq 1$ et $j=1,2$. Dans la suite, on s'intéresse surtout à $\lambda$ et on ne considère $\left(T_{n, j}\right)$ que dans la section 7 au travers de simulations numériques.

\section{Approximation BKW}

Le cas $x>x_{1}=1-b / a_{1}$. Avec $R_{0}>1$, on conjecture que $\lambda \rightarrow 0$ quand $N \rightarrow$ $+\infty$. On vérifiera a posteriori, comme l'ont fait par exemple Meerson et Sasorov (2008) pour un modèle différent, que c'est bien le cas. Le côté gauche de (6)(7) peut ainsi être négligé. Pour $x>x_{1}$, cherchons une approximation BKW (Bender et Orszag, 1978) de la forme

$$
\pi_{n, 1}=e^{-N S(x)-\Sigma(x)}, \quad \pi_{n, 2}=\frac{c(x)}{N} e^{-N S(x)-\Sigma(x)}
$$

où $x=n / N$. Un développement de Taylor à l'ordre 2 pour $S(x)$ et à l'ordre 1 pour $\Sigma(x)$ suggère que $\pi_{n \pm 1,1} \simeq e^{-N S(x) \mp S^{\prime}(x)-\frac{S^{\prime \prime}(x)}{2 N}-\Sigma(x) \mp \frac{\Sigma^{\prime}(x)}{N}}$. On obtient en remplaçant dans (6) et en simplifiant par $e^{-N S(x)-\Sigma(x)}$ que

$$
\begin{aligned}
0 \simeq & a_{1} N\left(x-\frac{1}{N}\right)\left(1-x+\frac{1}{N}\right) e^{S^{\prime}(x)-\frac{S^{\prime \prime}(x)}{2 N}+\frac{\Sigma^{\prime}(x)}{N}}-a_{1} N x(1-x) \\
& +b N\left(x+\frac{1}{N}\right) e^{-S^{\prime}(x)-\frac{S^{\prime \prime}(x)}{2 N}-\frac{\Sigma^{\prime}(x)}{N}}-b N x-q_{1}+q_{2} \frac{c(x)}{N} .
\end{aligned}
$$

Les termes d'ordre $N$ donnent

$$
0 \simeq N x\left[e^{S^{\prime}(x)}-1\right]\left[a_{1}(1-x)-b e^{-S^{\prime}(x)}\right] .
$$

Le premier terme entre crochets ne s'annule que pour les fonctions $S(x)$ constantes. Choisissons la solution annulant le second terme entre crochets, telle que $e^{S^{\prime}(x)}=$ $\frac{b}{a_{1}(1-x)}$, c'est-à-dire

$$
S(x)=s_{1}(x)=x \log \left(b / a_{1}\right)+x+(1-x) \log (1-x)
$$

à une constante additive près. Ceci implique que $S^{\prime \prime}(x)=\frac{1}{1-x}$. La fonction $\Sigma(x)$ s'obtient avec les termes d'ordre 1 :

$$
\begin{aligned}
0 \simeq & a_{1} N\left[x(1-x)+\frac{2 x-1}{N}\right] e^{S^{\prime}(x)}\left[1+\frac{S^{\prime \prime}(x)}{2 N}+\frac{\Sigma^{\prime}(x)}{N}\right]-a_{1} N x(1-x) \\
& +b N\left(x+\frac{1}{N}\right) e^{-S^{\prime}(x)}\left[1-\frac{S^{\prime \prime}(x)}{2 N}-\frac{\Sigma^{\prime}(x)}{N}\right]-b N x-q_{1} .
\end{aligned}
$$


En remplaçant $S(x)$ par son expression, on obtient

$$
\begin{aligned}
& \Sigma^{\prime}(x)=\frac{1}{x}-\frac{1 / 2}{1-x}+\frac{q_{1}}{a_{1}-b}\left(\frac{1}{x-x_{1}}-\frac{1}{x}\right), \\
& \Sigma(x)=\sigma_{1}(x)=\log \left(x \sqrt{1-x}\left[\frac{x-x_{1}}{x}\right]^{\frac{q_{1}}{a_{1}-b}}\right) .
\end{aligned}
$$

La fonction $c(x)$ intervenant dans $\pi_{n, 2}$ s'obtient à partir de (7), la forme retenue pour $\pi_{n, 2}$ étant choisie de manière à assurer la balance entre les différents termes. En effet, les termes d'ordre le plus élevé donnent

$$
0 \simeq \frac{c(x)}{N}\left\{N a_{2} x(1-x)\left[e^{S^{\prime}(x)}-1\right]+b N x\left(e^{-S^{\prime}(x)}-1\right)\right\}+q_{1} .
$$

En remplaçant $S(x)$ par son expression, on obtient

$$
c(x) \simeq \frac{q_{1}}{\left(a_{1}-a_{2}\right) x\left(x-x_{1}\right)} .
$$

En résumé, on peut conjecturer que pour $x>x_{1}$,

$$
\pi_{n, 1} \simeq k_{1} e^{-N s_{1}(x)-\sigma_{1}(x)}, \quad \pi_{n, 2} \simeq \frac{q_{1} \pi_{n, 1}}{N\left(a_{1}-a_{2}\right) x\left(x-x_{1}\right)},
$$

pour une certaine constante $k_{1}$. Considérons en particulier le comportement de $\pi_{n, 1}$ et de $\pi_{n, 2}$ pour $x>x_{1}$ et $x$ proche de $x_{1}$. Posons $c_{1}=b / a_{1}-1-\log \left(b / a_{1}\right)$. Comme $s_{1}^{\prime}\left(x_{1}\right)=0$, un développement de Taylor à l'ordre 2 suggère que $s_{1}(x) \simeq$ $s_{1}\left(x_{1}\right)+s_{1}^{\prime \prime}\left(x_{1}\right)\left(x-x_{1}\right)^{2} / 2 \simeq-c_{1}+\frac{a_{1}}{2 b}\left(x-x_{1}\right)^{2}$. Ainsi

$$
\pi_{n, 1} \simeq k_{1} \frac{e^{N c_{1}-N \frac{a_{1}}{2 b}\left(x-x_{1}\right)^{2}} x_{1}^{\frac{q_{1}}{a_{1}-b}-1}}{\sqrt{1-x_{1}}\left(x-x_{1}\right)^{\frac{q_{1}}{a_{1}-b}}}, \quad \pi_{n, 2} \simeq \frac{q_{1} \pi_{n, 1}}{N\left(a_{1}-a_{2}\right) x_{1}\left(x-x_{1}\right)} .
$$

Le cas $x<x_{2}=1-b / a_{2}$ lorsque $a_{1}>a_{2}>b$. Symétriquement, si $a_{2}>b$, cherchons une solution de la forme

$$
\pi_{n, 2}=e^{-N S(x)-\Sigma(x)}, \quad \pi_{n, 1}=\frac{c(x)}{N} e^{-N S(x)-\Sigma(x)}
$$

pour $x<x_{2}=1-b / a_{2}$. Le système (6)-(7) conduit, comme ci-dessus, à

$$
S(x)=s_{2}(x)=x \log \left(b / a_{2}\right)+x+(1-x) \log (1-x)
$$

à une constante additive près, tandis que

$$
\Sigma(x)=\sigma_{2}(x)=\log \left(x \sqrt{1-x}\left[\frac{x_{2}-x}{x}\right]^{\frac{q_{2}}{a_{2}-b}}\right), \quad c(x) \simeq \frac{q_{2}}{\left(a_{1}-a_{2}\right) x\left(x_{2}-x\right)} .
$$

En résumé, on peut conjecturer que pour $x<x_{2}$,

$$
\pi_{n, 2} \simeq k_{2} e^{-N s_{2}(x)-\sigma_{2}(x)}, \quad \pi_{n, 1} \simeq \frac{q_{2} \pi_{n, 2}}{N\left(a_{1}-a_{2}\right) x\left(x_{2}-x\right)},
$$


pour une certaine constante $k_{2}$. Pour $x<x_{2}$ et $x$ proche de $x_{2}$,

$$
\pi_{n, 2} \simeq k_{2} \frac{e^{N c_{2}-N \frac{a_{2}}{2 b}\left(x_{2}-x\right)^{2}} x_{2}^{\frac{q_{2}}{a_{2}-b}-1}}{\sqrt{1-x_{2}}\left(x_{2}-x\right)^{\frac{q_{2}}{a_{2}-b}}}, \quad \pi_{n, 1} \simeq \frac{q_{2} \pi_{n, 2}}{N\left(a_{1}-a_{2}\right) x_{2}\left(x_{2}-x\right)} .
$$

Les figures 2 et 3 montrent $-\left(\log \pi_{n, 1}\right) / N$ et $-\left(\log \pi_{n, 2}\right) / N$ en fonction de $n / N$ pour $1 \leq n \leq N$, avec $\pi_{n, i}$ calculé en utilisant le système (6)-(77). Les figures montrent aussi les approximations (10) et (12) en bleu foncé et rouge. La constante $k_{1}$ dans (10) est choisie de sorte que $\pi_{n, 1}$ coïncide avec son approximation pour $n=N-1$. La constante $k_{2}$ dans (12) est déterminée par $k_{1}$ comme cela est expliqué dans la section 5 ci-dessous.

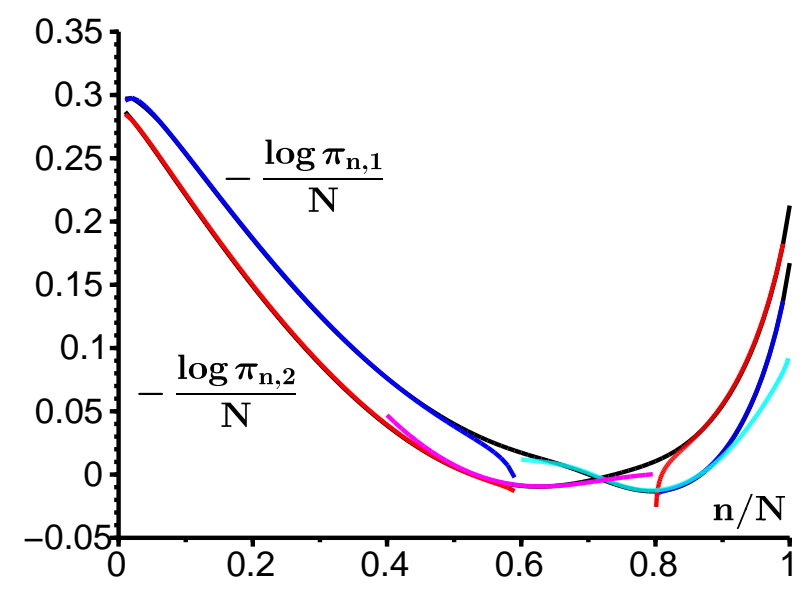

Figure 2 - L'exemple avec $a_{1}>a_{2}>b$. En noir, sous les autres courbes : $-\left(\log \pi_{n, 1}\right) / N$ et $-\left(\log \pi_{n, 2}\right) / N$ en fonction de $x=n / N$. Pour $\pi_{n, 1}$ : en bleu foncé, approximations BKW (10) pour $x>x_{1}$ et (12) pour $x<x_{2}$; en bleu clair, approximation diffusive (25) pour $x \simeq x_{1}$. Pour $\pi_{n, 2}$ : en rouge, approximations BKW (10) pour $x>x_{1}$ et (12) pour $x<x_{2}$; en rose, l'approximation diffusive (31) pour $x \simeq x_{2}$.

\section{Approximation dans la zone de variation lente}

Il reste à trouver une approximation de $\pi_{n, 1}$ et $\pi_{n, 2}$ pour $x_{2}<x<x_{1}$ lorsque $a_{1}>a_{2}>b$, et pour $0<x<x_{1}$ lorsque $a_{1}>b>a_{2}$. Sur ces intervalles, $S(x)=0$. Considérons le système (6)-(7) et essayons directement l'approximation continue $\pi_{n, 1} \simeq y(x), \pi_{n, 2} \simeq z(x)$, avec $x=n / N$. Un développement de

Taylor donne $\pi_{n \pm 1,1}=y(x \pm 1 / N) \simeq y(x) \pm \frac{y^{\prime}(x)}{N}+\frac{y^{\prime \prime}(x)}{2 N^{2}}$. Avec $\lambda \simeq 0$, cela 


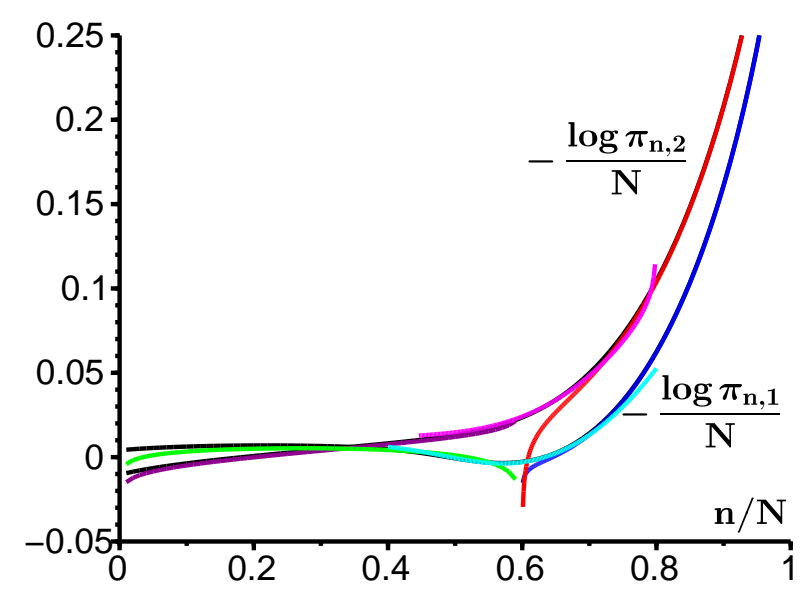

Figure 3 - L'exemple avec $a_{1}>b>a_{2}$. En noir, sous les autres courbes : $-\left(\log \pi_{n, 1}\right) / N$ et $-\left(\log \pi_{n, 2}\right) / N$ en fonction de $x=n / N$. Pour $\pi_{n, 1}$ : en bleu foncé, approximation BKW (10) pour $x>x_{1}$; en vert, approximation (20) pour $x<x_{1}$; en bleu clair, approximation diffusive (25) pour $x \simeq x_{1}$. Pour $\pi_{n, 2}$ : en rouge, approximation BKW (10) pour $x>x_{1}$; en violet, approximation (19) pour $x<x_{1}$; en rose, approximation diffusive (31) pour $x \simeq x_{1}$. 
conduit à l'approximation diffusive

$$
\begin{aligned}
0 & \simeq \frac{d}{d x}\left[\left(b x-a_{1} x(1-x)\right) y\right]-q_{1} y+q_{2} z+\frac{1}{2 N} \frac{d^{2}}{d x^{2}}\left[\left(a_{1} x(1-x)+b x\right) y\right] \\
0 & \simeq \frac{d}{d x}\left[\left(b x-a_{2} x(1-x)\right) z\right]-q_{2} z+q_{1} y+\frac{1}{2 N} \frac{d^{2}}{d x^{2}}\left[\left(a_{2} x(1-x)+b x\right) z\right] .
\end{aligned}
$$

En négligeant les termes de diffusion d'ordre $1 / N$, on obtient les équations

$$
\begin{aligned}
0 & \simeq \frac{d}{d x}\left[\left(b x-a_{1} x(1-x)\right) y(x)\right]-q_{1} y(x)+q_{2} z(x) \\
0 & \simeq \frac{d}{d x}\left[\left(b x-a_{2} x(1-x)\right) z(x)\right]-q_{2} z(x)+q_{1} y(x),
\end{aligned}
$$

qui sont de la même forme que les équations (29)-(30) de Slatkin (1978). En additionnant les équations (16) et (17), on trouve

$$
\frac{d}{d x}\left[\left(b x-a_{1} x(1-x)\right) y(x)+\left(b x-a_{2} x(1-x)\right) z(x)\right] \simeq 0 .
$$

La fonction entre crochets est donc constante. Comme $x$ est en facteur, cela suggère en faisant $x \rightarrow 0$ que cette constante est égale à zéro :

$$
\left(b x-a_{1} x(1-x)\right) y(x)+\left(b x-a_{2} x(1-x)\right) z(x) \simeq 0 .
$$

Cela permet d'exprimer $y(x)$ en fonction de $z(x)$. En remplaçant dans (17), on obtient après quelques manipulations que

$$
\begin{aligned}
\frac{1}{z(x)} \frac{d z}{d x} & \simeq \frac{q_{1}}{b x-a_{1} x(1-x)}+\frac{q_{2}}{b x-a_{2} x(1-x)}-\frac{b-a_{2}(1-2 x)}{b x-a_{2} x(1-x)}, \\
z(x) & \simeq \frac{C}{b x-a_{2} x(1-x)}\left(\frac{a_{1}(1-x)-b}{x}\right)^{\frac{q_{1}}{a_{1}-b}}\left(\frac{b-a_{2}(1-x)}{x}\right)^{\frac{q_{2}}{a_{2}-b}}
\end{aligned}
$$

pour une certaine constante $C$. Cette approximation est représentée en violet sur la figure 3, la constante $C$ étant déterminée par $k_{1}$ comme cela est indiqué dans la section 5 ci-dessous. Puis les équations (18) et (19) montrent que

$$
y(x) \simeq \frac{C}{a_{1} x(1-x)-b x}\left(\frac{a_{1}(1-x)-b}{x}\right)^{\frac{a_{1}}{a_{1}-b}}\left(\frac{b-a_{2}(1-x)}{x}\right)^{\frac{q_{2}}{a_{2}-b}} .
$$

Cette approximation est représentée en vert sur la figure 3. Lorsque $x$ est proche de $x_{1}$ avec $x<x_{1}$, on obtient

$$
y(x) \sim \frac{C}{a_{1} x_{1}}\left(\frac{a_{1}}{x_{1}}\right)^{\frac{a_{1}}{a_{1}-b}}\left(\frac{b-a_{2}\left(1-x_{1}\right)}{x_{1}}\right)^{\frac{q_{2}}{a_{2}-b}}\left(x_{1}-x\right)^{\frac{a_{1}}{a_{1}-b}-1} .
$$

Lorsque $a_{1}>b>a_{2}$, on trouve pour $x$ proche de 0

$$
\begin{aligned}
& y(x) \sim C\left(a_{1}-b\right)^{\frac{q_{1}}{a_{1}-b}-1}\left(b-a_{2}\right)^{\frac{q_{2}}{a_{2}-b}} x^{-1+\frac{q_{2}}{b-a_{2}}-\frac{q_{1}}{a_{1}-b}} \\
& z(x) \sim C\left(a_{1}-b\right)^{\frac{q_{1}}{a_{1}-b}}\left(b-a_{2}\right)^{\frac{q_{2}}{a_{2}-b}-1} x^{-1+\frac{q_{2}}{b-a_{2}}-\frac{q_{1}}{a_{1}-b}} .
\end{aligned}
$$


Rappelons que $R_{0}=\frac{a_{1} q_{2}+a_{2} q_{1}}{b\left(q_{1}+q_{2}\right)}>1$ équivaut à $\frac{q_{2}}{b-a_{2}}>\frac{q_{1}}{a_{1}-b}$ dans ce cas. En particulier, l'intégrale de $z(x)$ au voisinage de $0^{+}$est convergente. Si $a_{1}>a_{2}>b$, alors (19) montre que

$$
z(x) \simeq \frac{C}{a_{2} x_{2}}\left(\frac{a_{1}\left(1-x_{2}\right)-b}{x_{2}}\right)^{\frac{a_{1}}{a_{1}-b}}\left(\frac{a_{2}}{x_{2}}\right)^{\frac{q_{2}}{a_{2}-b}}\left(x-x_{2}\right)^{\frac{q_{2}}{a_{2}-b}-1}
$$

près de $x_{2}$ avec $x>x_{2}$.

\section{$5 \quad$ Approximation diffusive près de $x_{1}$ et $x_{2}$}

La situation près de $x_{1}$. Près de $x=x_{1}$ et $x=x_{2}$, le terme de diffusion dans (14)-(15) ne peut plus être négligé. L'analyse des zones de transition en $x_{1}$ et $x_{2}$ doit permettre de relier la constante $C$ intervenant dans (21) et (24) avec les constantes $k_{1}$ de (10) et $k_{2}$ de (12). Posons $x=x_{1}+\xi_{1} / \sqrt{N}$. Alors le coefficient de la dérivée d'ordre 1 dans (14) peut être approché par un développement de Taylor d'ordre 1, ce qui conduit à

$$
0 \simeq\left(a_{1}-b\right) \frac{d}{d \xi_{1}}\left[\xi_{1} y\right]-q_{1} y+q_{2} z+b\left(1-b / a_{1}\right) \frac{d^{2} y}{d \xi_{1}^{2}} .
$$

Le terme $q_{2} z$ est négligeable devant $q_{1} y$ lorsque $x \simeq x_{1}$, ce qui donne

$$
0 \simeq \frac{b}{a_{1}} \frac{d^{2} y}{d \xi_{1}^{2}}+\xi_{1} \frac{d y}{d \xi_{1}}+\left(1-\frac{q_{1}}{a_{1}-b}\right) y .
$$

Les changements de variable et d'inconnue

$$
\eta_{1}=\xi_{1} \sqrt{\frac{a_{1}}{2 b}}, \quad y\left(\eta_{1}\right)=e^{-\eta_{1}^{2}} Y\left(\eta_{1}\right)
$$

conduisent à l'équation différentielle de Hermite

$$
0 \simeq \frac{1}{2} \frac{d^{2} Y}{d \eta_{1}^{2}}-\eta_{1} \frac{d Y}{d \eta_{1}}-\frac{q_{1}}{a_{1}-b} Y
$$

Cherchant une solution sous la forme d'une série entière $Y\left(\eta_{1}\right)=\sum_{n=0}^{\infty} w_{n} \eta_{1}^{n}$, on obtient la relation de récurrence

$$
w_{n+2}=\frac{2\left(n+2 \alpha_{1}\right)}{(n+2)(n+1)} w_{n}
$$

où $\alpha_{1}=\frac{q_{1} / 2}{a_{1}-b}$. On a ainsi deux solutions linéairement indépendantes, l'une avec des puissances paires de $\eta_{1}$, l'autre avec des puissances impaires :

$$
Y\left(\eta_{1}\right)=\gamma_{1} \Phi\left(\alpha_{1}, \frac{1}{2} ; \eta_{1}^{2}\right)+\gamma_{2} \eta_{1} \Phi\left(\alpha_{1}+\frac{1}{2}, \frac{3}{2} ; \eta_{1}^{2}\right),
$$


où $\gamma_{1}$ et $\gamma_{2}$ sont des constantes et

$$
\Phi(\alpha, \beta ; z)=\sum_{n=0}^{\infty} \frac{\alpha^{(n)} z^{n}}{\beta^{(n)} n !}, \quad \alpha^{(0)}=1, \quad \alpha^{(n)}=\alpha(\alpha+1)(\alpha+2) \cdots(\alpha+n-1)
$$

est la fonction hypergéometrique confluente de Kummer. D'après Maroni (1997, Eq. (93)), on a $\Phi(\alpha, \beta ; z) \sim \frac{\Gamma(\beta)}{\Gamma(\alpha)} e^{z} z^{\alpha-\beta}$ quand $z \rightarrow+\infty$, où $\Gamma$ est la fonction Gamma d'Euler. Ainsi

$$
Y\left(\eta_{1}\right) \sim e^{\eta_{1}^{2}}\left|\eta_{1}\right|^{2 \alpha_{1}-1}\left(\frac{\gamma_{1} \Gamma\left(\frac{1}{2}\right)}{\Gamma\left(\alpha_{1}\right)}-\frac{\gamma_{2} \Gamma\left(\frac{3}{2}\right)}{\Gamma\left(\alpha_{1}+\frac{1}{2}\right)}\right), \quad \eta \rightarrow-\infty
$$

Donc $y\left(\eta_{1}\right)=e^{-\eta_{1}^{2}} Y\left(\eta_{1}\right)$ a le même comportement asymptotique que (21) lorsque $\eta_{1} \rightarrow-\infty$, pourvu que

$$
\left[\sqrt{\frac{N a_{1}}{2 b}}\right]^{\frac{q_{1}}{a_{1}-b}-1}\left(\frac{\gamma_{1} \Gamma\left(\frac{1}{2}\right)}{\Gamma\left(\alpha_{1}\right)}-\frac{\gamma_{2} \Gamma\left(\frac{3}{2}\right)}{\Gamma\left(\alpha_{1}+\frac{1}{2}\right)}\right)=\frac{C\left(b-a_{2}\left(1-x_{1}\right)\right)^{\frac{q_{2}}{a_{2}-b}}}{a_{1}^{1-\frac{q_{1}}{a_{1}-b}} x_{1}^{1+\frac{q_{1}}{a_{1}-b}+\frac{q_{2}}{a_{2}-b}}} .
$$

Quand au contraire $\eta_{1} \rightarrow+\infty$, (11) suggère que $Y\left(\eta_{1}\right)$ doit se comporter comme une certaine constante multipliée par $\eta_{1}^{-2 \alpha_{1}}$. La théorie des fonctions hypergéométriques confluentes (Maroni, 1997) montre qu'il faut imposer

$$
\gamma_{1}=\frac{\Gamma\left(\frac{1}{2}\right)}{\Gamma\left(\alpha_{1}+\frac{1}{2}\right)} K, \quad \gamma_{2}=\frac{\Gamma\left(-\frac{1}{2}\right)}{\Gamma\left(\alpha_{1}\right)} K
$$

pour une certaine constante $K$ pour obtenir un tel comportement asymptotique. En effet, on a alors

$$
Y\left(\eta_{1}\right)=K\left(\frac{\Gamma\left(\frac{1}{2}\right)}{\Gamma\left(\alpha_{1}+\frac{1}{2}\right)} \Phi\left(\alpha_{1}, \frac{1}{2} ; \eta_{1}^{2}\right)+\frac{\Gamma\left(-\frac{1}{2}\right)}{\Gamma\left(\alpha_{1}\right)} \eta_{1} \Phi\left(\alpha_{1}+\frac{1}{2}, \frac{3}{2} ; \eta_{1}^{2}\right)\right),
$$

c'est-à-dire $Y\left(\eta_{1}\right)=K \Psi\left(\alpha_{1}, \frac{1}{2} ; \eta_{1}^{2}\right)$ pour $\eta_{1}>0$, où $\Psi(\alpha, \beta ; z)$ est la fonction hypergéometrique confluente de Tricomi définie par

$$
\Psi(\alpha, \beta ; z)=\frac{\Gamma(1-\beta)}{\Gamma(\alpha-\beta+1)} \Phi(\alpha, \beta ; z)+\frac{\Gamma(\beta-1)}{\Gamma(\alpha)} z^{1-\beta} \Phi(\alpha-\beta+1,2-\beta ; z) .
$$

D'après Maroni (1997, Eq. (90)), on a bien $\Psi(\alpha, \beta ; z) \sim z^{-\alpha}$ quand $z \rightarrow+\infty$. Donc $Y\left(\eta_{1}\right) \sim K \eta_{1}^{-2 \alpha_{1}}$ quand $\eta_{1} \rightarrow+\infty$. L'équivalent de $e^{-\eta_{1}^{2}} Y\left(\eta_{1}\right)$ coïncide alors avec (11) pourvu que

$$
K\left[\sqrt{\frac{N a_{1}}{2 b}}\right]^{-\frac{q_{1}}{a_{1}-b}}=k_{1} \frac{e^{N c_{1}}}{\sqrt{1-x_{1}}} x_{1}^{\frac{q_{1}}{a_{1}-b}-1} .
$$

En résumé, les relations (26), (27) et (29) déterminent $K, \gamma_{1}, \gamma_{2}$ et $C$ en fonction de $k_{1}$. En particulier, puisque $\Gamma\left(-\frac{1}{2}\right)=-2 \sqrt{\pi}, \Gamma\left(\frac{1}{2}\right)=\sqrt{\pi}$ et $\Gamma\left(\frac{3}{2}\right)=\frac{\sqrt{\pi}}{2}$, 
l'équation (26) donne

$$
\left[\sqrt{\frac{N a_{1}}{2 b}}\right]^{\frac{q_{1}}{a_{1}-b}-1} \frac{2 \pi K}{\Gamma\left(\alpha_{1}\right) \Gamma\left(\alpha_{1}+\frac{1}{2}\right)}=\frac{C\left(b-a_{2}\left(1-x_{1}\right)\right)^{\frac{q_{2}}{a_{2}-b}}}{a_{1}^{1-\frac{q_{1}}{a_{1}-b}} x_{1}^{1+\frac{q_{1}}{a_{1}-b}+\frac{q_{2}}{a_{2}-b}}} .
$$

Une forme alternative peut être obtenue en utilisant la formule de LegendreGauss $\Gamma\left(\alpha_{1}\right) \Gamma\left(\alpha_{1}+\frac{1}{2}\right)=\frac{\sqrt{\pi}}{2^{2 \alpha_{1}-1}} \Gamma\left(2 \alpha_{1}\right)$.

La situation près de $x_{2}$. Lorsque $a_{2}>b$, une analyse similaire peut être faite près de $x=x_{2}=1-b / a_{2}$ en partant de (15) et en négligeant le terme $q_{1} y$ devant $q_{2} z$. Posons $x=x_{2}+\frac{\xi_{2}}{\sqrt{N}}, \eta_{2}=\xi_{2} \sqrt{\frac{a_{2}}{2 b}}, z\left(\eta_{2}\right)=e^{-\eta_{2}^{2}} Z\left(\eta_{2}\right)$, et $\alpha_{2}=\frac{q_{2} / 2}{a_{2}-b}$. Alors

$$
Z\left(\eta_{2}\right)=\delta_{1} \Phi\left(\alpha_{2}, \frac{1}{2} ; \eta_{2}^{2}\right)+\delta_{2} \eta_{2} \Phi\left(\alpha_{2}+\frac{1}{2}, \frac{3}{2} ; \eta_{2}^{2}\right)
$$

où $\delta_{1}$ et $\delta_{2}$ sont des constantes. Le comportement pour $\eta_{2} \rightarrow+\infty$ coïncide avec (24) pourvu que

$$
\left[\sqrt{\frac{N a_{2}}{2 b}}\right]^{\frac{q_{2}}{a_{2}-b}-1}\left(\frac{\delta_{1} \Gamma\left(\frac{1}{2}\right)}{\Gamma\left(\alpha_{2}\right)}+\frac{\delta_{2} \Gamma\left(\frac{3}{2}\right)}{\Gamma\left(\alpha_{2}+\frac{1}{2}\right)}\right)=\frac{C\left[a_{1}\left(1-x_{2}\right)-b\right]^{\frac{q_{1}}{a_{1}-b}}}{a_{2}^{1-\frac{q_{2}}{a_{2}-b}} x_{2}^{1+\frac{q_{1}}{a_{1}-b}+\frac{q_{2}}{a_{2}-b}}} .
$$

Posons

$$
\delta_{1}=\frac{\Gamma\left(\frac{1}{2}\right)}{\Gamma\left(\alpha_{2}+\frac{1}{2}\right)} \hat{K}, \quad \delta_{2}=-\frac{\Gamma\left(-\frac{1}{2}\right)}{\Gamma\left(\alpha_{2}\right)} \hat{K}
$$

pour une certaine constante $\hat{K}$ (noter le signe moins dans $\delta_{2}$ ). Alors $Z\left(\eta_{2}\right)=$ $\hat{K} \Psi\left(\alpha_{2}, \frac{1}{2} ; \eta_{2}^{2}\right)$ quand $\eta_{2}<0$. Donc $e^{-\eta_{2}^{2}} Z\left(\eta_{2}\right) \sim \hat{K} e^{-\eta_{2}^{2}}\left|\eta_{2}\right|^{-2 \alpha_{2}}$ pour $\eta_{2} \rightarrow-\infty$. Ceci cö̈ncide avec (13) pourvu que

$$
\hat{K}\left[\sqrt{\frac{N a_{2}}{2 b}}\right]^{-\frac{q_{2}}{a_{2}-b}}=k_{2} \frac{e^{N c_{2}}}{\sqrt{1-x_{2}}} x_{2}^{\frac{q_{2}}{a_{2}-b}-1} .
$$

Comme ci-dessus, (32) et (33) donnent

$$
\left[\sqrt{\frac{N a_{2}}{2 b}}\right]^{\frac{q_{2}}{a_{2}-b}-1} \frac{2 \pi \hat{K}}{\Gamma\left(\alpha_{2}\right) \Gamma\left(\alpha_{2}+\frac{1}{2}\right)}=\frac{C\left[a_{1}\left(1-x_{2}\right)-b\right]^{\frac{q_{1}}{a_{1}-b}}}{a_{2}^{1-\frac{q_{2}}{a_{2}-b}} x_{2}^{1+\frac{q_{1}}{a_{1}-b}+\frac{q_{2}}{a_{2}-b}}} .
$$

Ainsi les constantes $k_{2}, \hat{K}, \delta_{1}$ et $\delta_{2}$ sont déterminées par la constante $C$.

\section{La valeur propre}

Rappelons que $M \pi=\lambda \pi$. En additionnant toutes les lignes de ce système d'équations, on obtient $\lambda \sum_{n=0}^{N}\left(\pi_{n, 1}+\pi_{n, 2}\right)=0$. Or $\lambda<0$. Donc $\sum_{n=0}^{N}\left(\pi_{n, 1}+\right.$ $\left.\pi_{n, 2}\right)=0$. Les deux premières lignes du système sont

$$
\begin{aligned}
& \lambda \pi_{0,1}=-q_{1} \pi_{0,1}+q_{2} \pi_{0,2}+b \pi_{1,1}, \\
& \lambda \pi_{0,2}=-q_{2} \pi_{0,2}+q_{1} \pi_{0,1}+b \pi_{1,2} .
\end{aligned}
$$


En les additionnant, on trouve

$$
\lambda=b \frac{\pi_{1,1}+\pi_{1,2}}{\pi_{0,1}+\pi_{0,2}}=-b \frac{\pi_{1,1}+\pi_{1,2}}{\sum_{n=1}^{N}\left(\pi_{n, 1}+\pi_{n, 2}\right)} .
$$

Considérons d'abord le cas où $a_{1}>b>a_{2}$ avec $R_{0}>1$. Alors (19) montre que

$$
\frac{1}{N} \sum_{n=1}^{N} \pi_{n, 2} \simeq \int_{0}^{x_{1}} z(x) d x \simeq \kappa_{1} C
$$

où $\kappa_{1}$ est une constante positive indépendante de $N$. En utilisant le fait que le pic de $\pi_{n, 1}$ est proche de $x=n / N=x_{1}$ et les relations (25), (28) et (30), on trouve

$$
\frac{1}{N} \sum_{n=1}^{N} \pi_{n, 1} \simeq \int_{-\infty}^{+\infty} y\left(\eta_{1}\right) d \eta_{1} \frac{d x}{d \eta_{1}} \simeq \kappa_{2} \frac{K}{\sqrt{N}} \simeq \kappa_{3} C N^{-\frac{q_{1} / 2}{a_{1}-b}},
$$

où les $\kappa_{j}$ (ci-dessus et ci-dessous) sont encore des constantes positives indépendantes de $N$. Ainsi le terme dominant pour $N$ grand dans le dénominateur de (36) est celui impliquant $\pi_{n, 2}$. Comme au numérateur $\pi_{1,1}$ et $\pi_{1,2}$ peuvent être approchés par $y\left(\frac{1}{N}\right)$ et $z\left(\frac{1}{N}\right)$ où $y(x)$ et $z(x)$ sont donnés par (22)-(23), on obtient que

$$
\lambda \simeq-\kappa_{4} \frac{\left(\frac{1}{N}\right)^{\frac{q_{2}}{b-a_{2}}-\frac{q_{1}}{a_{1}-b}-1}}{N} \simeq-\kappa_{4} N^{-\frac{q_{2}}{b-a_{2}}+\frac{q_{1}}{a_{1}-b}} .
$$

Autrement dit, $-1 / \lambda$ croît avec $N$ comme une loi de puissance d'exposant $\omega=\frac{q_{2}}{b-a_{2}}-\frac{q_{1}}{a_{1}-b}>0$, ainsi qu'annoncé dans l'introduction. On remarque d'ailleurs que $\omega$ tend vers $+\infty$ lorsque $a_{2}$ tend vers $b$ par valeurs inférieures.

Considérons maintenant le cas où $a_{1}>a_{2}>b$. Pour simplifier, introduisons la notation $f \approx g$ (à ne pas confondre avec le symbole informel $\simeq$ utilisé cidessus) si $(\log f) / N-(\log g) / N \rightarrow 0$ quand $N \rightarrow+\infty$. En particulier $N^{\beta} \approx$ 1 pour tout $\beta$. Alors (12) avec $x=1 / N$ montre que $\pi_{1,1} \approx \pi_{1,2}$. De plus, $\pi_{1,2} \approx k_{2} e^{-N s_{2}(1 / N)} \approx k_{2} e^{-s_{2}^{\prime}(0)} \approx k_{2}$. Par ailleurs, le pic de $\pi_{n, 1}$ est proche de $x=n / N=x_{1}$ alors que celui de $\pi_{n, 2}$ est proche de $x_{2}$. Ainsi (37) est encore valable et montre que $\frac{1}{N} \sum_{n=1}^{N} \pi_{n, 1} \approx C$, tandis que (31), (33) et (35) donnent

$$
\frac{1}{N} \sum_{n=1}^{N} \pi_{n, 2} \simeq \int_{-\infty}^{+\infty} z\left(\eta_{2}\right) d \eta_{2} \frac{d x}{d \eta_{2}} \simeq \kappa_{5} \frac{\hat{K}}{\sqrt{N}} \simeq \kappa_{5} C N^{-\frac{q_{2} / 2}{a_{2}-b}} \approx C .
$$

Mais (34) et (35) montrent que $C \approx \hat{K} \approx k_{2} e^{N c_{2}}$. Ainsi (36) donne $-\lambda \approx e^{-N c_{2}}$ et $-1 / \lambda \approx e^{N c_{2}}$. Finalement, $-1 / \lambda$ croît à peu près exponentiellement avec un paramètre $c_{2}$. 


\section{Le temps moyen jusqu'à extinction}

Le système linéaire (8)-(9) peut être résolu avec la méthode numérique présentée par Artalejo et al. (2013). Elle n'utilise que des matrices carrées d'ordre 2 et réduit l'instabilité numérique. Rappelons que $q_{1}=q_{2}=1$ et que $b=2$.

Premier exemple : $a_{1}>a_{2}>b$. Prenons $a_{1}=10$ et $a_{2}=5$. La figure 4a montre $T_{n, 1}$ et $T_{n, 2}$ en fonction de $x=n / N$. La figure $4 \mathrm{~b}$ suggère que le temps moyen jusqu'à extinction, partant par exemple de $N$ personnes infectées dans l'environnement 1 , croît à peu près exponentiellement : $T_{N, 1} \approx e^{c N}$. Une régression linéaire donne $c \simeq 0.32$. Noter que $c_{1} \simeq 0.81$ et $c_{2} \simeq 0.32$, ce qui suggère bien que $c=c_{2}$.
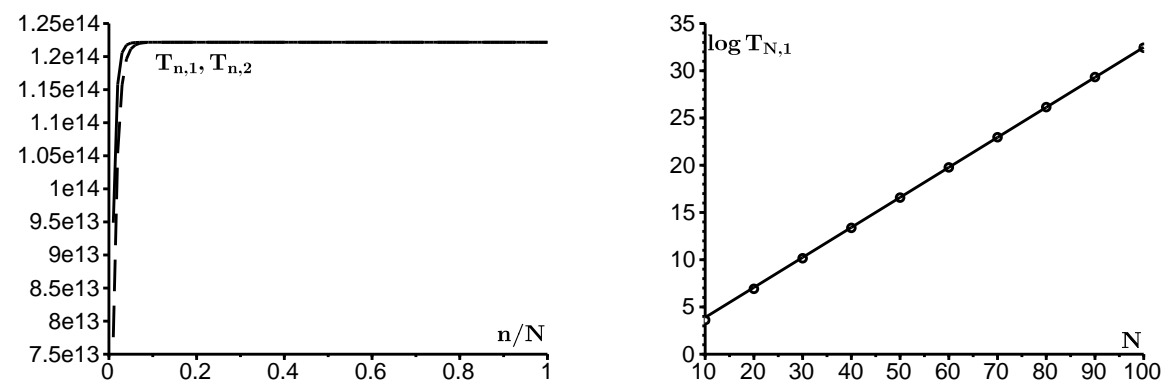

Figure 4 - Le cas $a_{1}>a_{2}>b$. a) $T_{n, 1}$ [ligne pleine] et $T_{n, 2}$ [ligne pointillée] en fonction de $n / N$ lorsque $N=100$. b) $\log T_{N, 1}$ en fonction de $N$.

Deuxième exemple : $a_{1}>b>a_{2}$ et $R_{0}>1$. Prenons $a_{1}=5$ et $a_{2}=1$. Donc $R_{0}=1.5>1$. La figure 5 a montre $T_{n, 1}$ et $T_{n, 2}$ en fonction de $x=n / N$. La figure $5 \mathrm{~b}$ montre $\log T_{N, 1}$ en fonction de $\log N$. On obtient une ligne droite suggérant une loi de puissance $T \sim \kappa N^{\omega}$. La pente, obtenue par régression linéaire, est $\omega \simeq 0.67$. Dans ce cas, on a $\frac{q_{2}}{b-a_{2}}-\frac{q_{1}}{a_{1}-b}=1-\frac{1}{3}=\frac{2}{3}$, ce qui correspond bien à la pente mesurée.

\section{Remarques}

Comparaison avec le cas périodique. Pour mettre en évidence la différence entre un environnement aléatoire et un environnement périodique, reprenons le cas où $a_{1}=5$ et $a_{2}=1$, tandis que $q_{1}=q_{2}=1$ et $b=2$. Alors $u_{1}=u_{2}=0.5$ : l'environnement passe en moyenne la moitié du temps dans l'état 1, l'autre moitié dans l'état 2. La figure 6 remontre d'un côté comment $T_{N, 1}$ croît en fonction de $N$ comme une loi de puissance. D'un autre côté, considérons le même modèle SIS mais dans un environnement $T$-périodique (Bacaër, 2015) : 

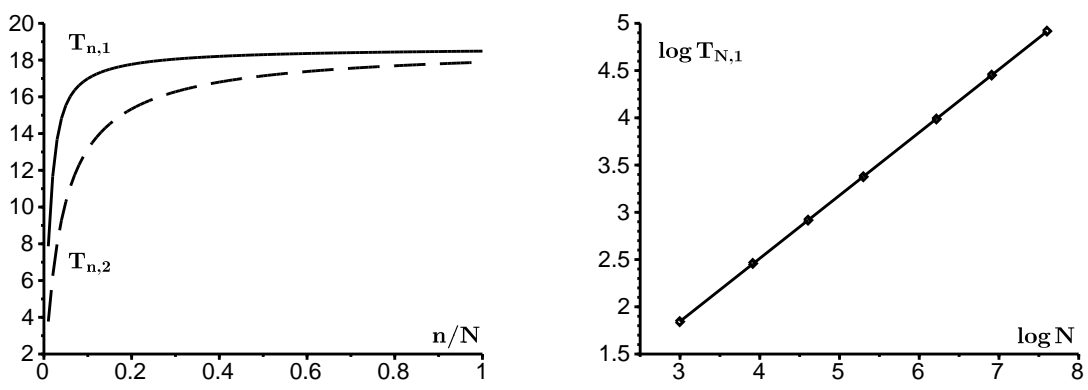

Figure 5 - Le cas $a_{1}>b>a_{2}$ avec $R_{0}>1$. a) $T_{n, 1}$ et $T_{n, 2}$ en fonction de $x=n / N$ lorsque $N=100$. b) $\log T_{N, 1}$ en fonction de $\log N$.

choisissons $T=\frac{1}{q_{1}}+\frac{1}{q_{2}}, a(t)=a_{1}$ pour $0<t<u_{1} T$, et $a(t)=a_{2}$ pour $u_{1} T<$ $t<T$. Pour ce modèle, soit $e^{\Lambda T}$ le multiplicateur de Floquet associé au processus d'extinction. La figure 6 montre comment $-1 / \Lambda$ croît exponentiellement avec $N$.

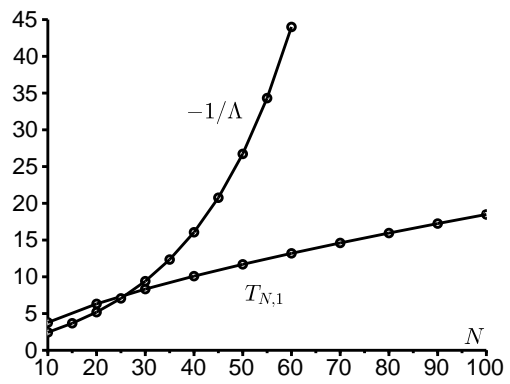

FIgURE 6 - Le temps moyen jusqu'à extinction $T_{N, 1}$ dans un environnement aléatoire et $-1 / \Lambda$ dans un environnement périodique ressemblant.

Les équations de champ moyen. Il n'est peut-être pas inutile de rappeler suivant Bacaër et Ed-Darraz (2014) que les équations de champ moyen

$$
\begin{aligned}
& \frac{d I_{1}}{d t}=a_{1} I_{1}\left(1-\frac{I_{1}}{N}\right)-b I_{1}-q_{1} I_{1}+q_{2} I_{2} \\
& \frac{d I_{2}}{d t}=a_{2} I_{2}\left(1-\frac{I_{2}}{N}\right)-b I_{2}-q_{2} I_{2}+q_{1} I_{1},
\end{aligned}
$$


une fois linéarisées près de l'état d'équilibre $(0,0)$, conduisent à la matrice jacobienne

$$
\left(\begin{array}{cc}
a_{1}-b-q_{1} & q_{2} \\
q_{1} & a_{2}-b-q_{2}
\end{array}\right) .
$$

Mais cette matrice peut avoir une valeur propre strictement positive, de sorte que l'équilibre trivial est instable, alors que $R_{0}<1$.

Autre limite. Les résultats obtenus considèrent que les paramètres $q_{j}, a_{j}$, et $b$ sont fixés tandis que $N$ tend vers l'infini. Les choses sont évidemment différentes si par exemple on laisse aussi $q_{1}$ et $q_{2}$ tendre vers l'infini avec un rapport $q_{1} / q_{2}$ fixé. Le modèle se rapproche alors d'un modèle homogénéisé en environnement constant avec un paramètre de contact moyenné $\bar{a}=a_{1} u_{1}+a_{2} u_{2}$.

Le cas sous-critique. On n'a pas abordé le cas sous-critique $R_{0}<1$ avec $a_{1}>b>a_{2}$ ou $b>a_{1}>a_{2}$. Le comportement du temps moyen jusqu'à extinction est alors sensiblement le même que dans un environnement constant : partant d'une fraction fixe de la population qui est infectée, le temps moyen est de l'ordre de $\log N$ (Doering et al., 2005). Partant d'une fraction infectée telle que $1 / N$, le temps moyen tend au contraire vers une limite. Dans le cas d'un environnement aléatoire, c'est aussi ce que suggèrent les simulations numériques.

\section{Chaîne de Markov avec un nombre fini d'états}

Avec $J$ états, rappelons que le générateur infinitésimal $Q$ est tel que $Q_{j, j}=$ $-\sum_{i=1}^{J} Q_{i, j}$ pour tout $j$. Supposons que cette matrice $Q$ soit irréductible et que $a_{1}>a_{2}>\cdots>a_{J}$. On peut conjecturer que si $a_{J}>b$, alors le temps moyen jusqu'à extinction croît à peu près exponentiellement avec la taille $N$ de la population avec un taux $c_{J}=b / a_{J}-1-\log \left(b / a_{J}\right)$. C'est en effet dans l'environnement $J$ que l'extinction est la plus probable.

Si en revanche $b>a_{J}$ et $R_{0}>1$, notons $\pi_{n, j} \simeq y_{j}(x)$ avec $x=n / N$. Au voisinage de $x=0$, le système (16)-(17) devient

$$
0 \simeq\left(b-a_{j}\right) \frac{d}{d x}\left(x y_{j}\right)+\sum_{k=1}^{J} Q_{j, k} y_{k} .
$$

En cherchant une solution de la forme $y_{j}=\theta_{j} x^{\omega-1}$ avec $\omega>0$ et $\theta_{j}>0$ pour tout $j$, on trouve

$$
0 \simeq \omega\left(b-a_{j}\right) \theta_{j}+\sum_{k=1}^{J} Q_{j, k} \theta_{k}
$$

pour $1 \leq j \leq J$. Avec les notations de l'introduction, on a donc le problème de valeur propre généralisé $Q \theta=\omega(A-B) \theta$, avec $\theta=\left(\theta_{1}, \ldots, \theta_{J}\right)$. Il s'agit de montrer qu'il existe un unique nombre $\omega>0$ tel que ce problème ait une solution avec $\theta_{j}>0$ pour tout $j$. Posons $D=A-B$. C'est une matrice diagonale. 
Pour tout nombre réel $s$, rappelons que $\mu(s)=\max \{\Re(\lambda) ; \lambda \in \sigma(Q-s D)\}$. La matrice $Q-s D$ a tous ses coefficients en dehors de la diagonale qui sont positifs ou nuls; de plus, cette matrice est irréductible. D'après un corollaire du théorème de Perron et Frobenius (Smith, 1995, Corollaire 3.2), $\mu(s)$ est une valeur propre simple de $Q-s D$ (appelée valeur propre dominante) et il existe un vecteur propre $\phi(s)$ dont toutes les composantes sont strictement positives tel que $(Q-s D) \phi(s)=\mu(s) \phi(s)$. Ce vecteur propre est unique avec la normalisation $\sum_{j=1}^{J} \phi_{j}(s)=1$. D'après le même corollaire, l'existence d'un vecteur $\theta$ tel que $\theta_{j}>0$ pour tout $j$ et $(Q-\omega D) \theta=0$ équivaut en fait à $\mu(\omega)=0$. Il s'agit donc de montrer que l'équation $\mu(\omega)=0$ avec $\omega>0$ a une unique solution.

Concernant l'existence, on remarque d'abord que le générateur infinitésimal $Q$ correspond à $s=0$ : on a $\mu(0)=0$ et l'on note $u=\phi(0)$ le vecteur propre à droite. Le vecteur ligne $\mathbf{1}=[1, \ldots, 1]$ est un vecteur propre à gauche de $Q$ associé à la valeur propre 0 . Ainsi $\mathbf{1} u=\sum_{j=1}^{J} u_{j}=1$. D'après le théorème de perturbation des valeurs propres simples, la fonction $\mu(s)$ est dérivable en $s=0$ et $\mu^{\prime}(0)=-\mathbf{1} D u$. Or $R_{0}>1$ équivaut à $\mathbf{1} D u=\sum_{j=1}^{J}\left(a_{j}-b\right) u_{j}>0$. Donc $\mu^{\prime}(0)<0$. Par ailleurs, on a $(Q / s-D) \phi(s)=(\mu(s) / s) \phi(s)$ pour tout $s>0$. Lorsque $s \rightarrow+\infty$, on a $Q / s-D \rightarrow-D$. Par continuité du spectre, $\mu(s) / s \rightarrow \max _{j}\left(-D_{j, j}\right)=\max _{j}\left(b-a_{j}\right)=b-a_{J}>0$. Donc $\mu(s) \rightarrow+\infty$ quand $s \rightarrow+\infty$. Comme $\mu(0)=0$ et $\mu^{\prime}(0)<0$, on en déduit qu'il existe $\omega>0$ tel que $\mu(\omega)=0$.

Quant à l'unicité, remarquons tout d'abord que la fonction $\mu(s)$ est convexe. En effet, pour tous les nombres réels $\omega_{1}, \omega_{2}$, et $0<\varepsilon<1$, on voit que $\mu\left(\varepsilon \omega_{1}+\right.$ $\left.(1-\varepsilon) \omega_{2}\right)$ est la valeur propre dominante de la matrice $Q-\varepsilon \omega_{1} D-(1-\varepsilon) \omega_{2} D$. D'après un théorème de Cohen (1981), cette valeur propre est inférieure ou égale à $\varepsilon \mu\left(\omega_{1}\right)+(1-\varepsilon) \mu\left(\omega_{2}\right)$. Ceci prouve la convexité. Supposons maintenant qu'il existe $\omega_{1}>\omega_{2}>0$ tels que $\mu\left(\omega_{1}\right)=\mu\left(\omega_{2}\right)=0$. Comme $\mu(0)=0$ et $\mu(s)$ est convexe, on en déduit que $\mu(s)=0$ pour tout $0 \leq s \leq \omega_{1}$. Ceci contredit le fait que $\mu^{\prime}(0)<0$. CQFD.

Lorsque $a_{j} \neq b$ pour tout $j$, la matrice $A-B$ est inversible. Ainsi $Q \theta=$ $\omega(A-B) \theta$ équivaut à $(A-B)^{-1} Q \theta=\omega \theta$. Donc $\omega$ est la valeur propre de $(A-B)^{-1} Q$ dont la partie réelle est la plus grande.

Enfin l'équation (36) devient

$$
\lambda=-b \frac{\sum_{j=1}^{J} \pi_{1, j}}{\sum_{j=1}^{J} \sum_{n=1}^{N} \pi_{n, j}} .
$$

Au numérateur, on a $\pi_{1, j} \simeq \theta_{j}(1 / N)^{\omega-1}$. Au dénominateur, on a $\sum_{n=1}^{N} \pi_{n, j} \simeq$ $N \theta_{j} \int_{0}^{1} x^{\omega-1} d x$. Donc $-1 / \lambda$ semble bien croître avec $N$ comme $N^{\omega}$.

Il est sans doute possible de généraliser un tel résultat au cas où l'environnement est gouverné par exemple par une équation différentielle stochastique $d \xi=f(\xi(t))+\sigma d B(t)$. Soit $L^{*}$ l'opérateur différentiel défini par $\left(L^{*} u\right)(\xi)=$ $\frac{\sigma^{2}}{2} \frac{d^{2} u}{d \xi^{2}}-\frac{d}{d \xi}(f(\xi) u(\xi))$. Supposons que $u(\xi)$ soit une probabilité invariante : $L^{*} u=0, u>0$ et $\int_{-\infty}^{+\infty} u(\xi) d \xi=1$. Ainsi, dans le cas de l'équation de Lange- 
vin avec $f(\xi)=-k \xi$ et $k>0$, on a $u(\xi)=\sqrt{\frac{k}{\pi \sigma^{2}}} e^{-k \xi^{2} / \sigma^{2}}$. Supposons que le contact $a(\cdot)$ soit une fonction sur $\mathbb{R}$ telle que min $a(\cdot)<b$ et

$$
R_{0}=\left(\lim _{T \rightarrow \infty} \frac{1}{T} \int_{0}^{T} a(\xi(t)) d t\right) / b=\left(\int_{-\infty}^{+\infty} a(\xi) u(\xi) d \xi\right) / b>1 .
$$

On conjecture alors que le temps moyen d'extinction croît avec $N$ comme $N^{\omega}$, où $\omega$ est l'unique nombre positif tel que l'opérateur différentiel $L^{*}-\omega[a(\cdot)-b]$ ait une valeur propre principale égale à 0 . Pour la convexité de cette valeur propre en fonction de $\omega$, voir Kato (1982). Noter que l'adjoint de $L^{*}$ est $L=\frac{\sigma^{2}}{2} \frac{d^{2}}{d \xi^{2}}+f(\xi) \frac{d}{d \xi}$ et que $L \mathbf{1}=0$ si 1 désigne la fonction constante égale à 1 .

\section{Un environnement à la fois périodique et aléatoire}

Revenons au cas d'un ensemble fini d'environnements et supposons désormais que le générateur infinitésimal $Q(t)$ soit une fonction périodique de période $\mathcal{T}$, continue ou continue par morceaux, et irréductible au moins sur un intervalle de $t$. Le système (4)-(5) prend la forme $\frac{d P}{d t}=M(t) P$. Suivant Bacaër (2015), il existe alors un unique couple $(\lambda, \pi)$ tel que $\lambda<0$, tel que $\pi(t)=$ $\left(\pi_{n, j}(t)\right)_{0 \leq n \leq N, 1 \leq j \leq J}$ soit une fonction $\mathcal{T}$-périodique, tel que $\lambda \pi(t)+\frac{d \pi}{d t}=$ $M(t) \pi(t)$, tel que $\frac{1}{N} \sum_{n=1}^{N} \sum_{j=1}^{J} \pi_{n, j}(t)=1$, et tel que $\pi_{n, j}(t)>0$ pour $1 \leq n \leq$ $N$ et $1 \leq j \leq J$. De plus, $\sum_{n=0}^{N} \sum_{j=1}^{J} \pi_{n, j}(t)=0$. Il existe aussi une unique fonction $\mathcal{T}$-périodique $u(t)$ telle que $\frac{d u}{d t}=Q(t) u(t), u_{j}(t)>0$, et $\sum_{j=1}^{J} u_{j}(t)=1$. Suivant Bacaër et Ed-Darraz (2014), on pose $R_{0}=\left(\frac{1}{\mathcal{T}} \int_{0}^{\mathcal{T}} \sum_{j=1}^{J} a_{j} u_{j}(t) d t\right) / b$.

On conjecture que si $a_{1}>\ldots>a_{J}>b$, alors le temps moyen jusqu'à extinction croît encore exponentiellement avec $N$ avec un taux $c_{J}=b / a_{J}-$ $1-\log \left(b / a_{J}\right)$. Si en revanche $a_{J}<b$ mais $R_{0}>1$, l'approximation continue $\pi_{n, j}(t) \simeq y_{j}(t, x)$ conduit à généraliser (38) au voisinage de $x=0$ par

$$
\frac{\partial y_{j}}{\partial t} \simeq\left(b-a_{j}\right) \frac{\partial}{\partial x}\left(x y_{j}\right)+\sum_{k=1}^{J} Q_{j, k}(t) y_{k} .
$$

Une solution de la forme $y_{j}(t, x)=\theta_{j}(t) x^{\omega-1}$, avec une fonction $\theta(t)=\left(\theta_{j}(t)\right)$ qui est $\mathcal{T}$-périodique, doit vérifier

$$
\frac{d \theta}{d t}=(Q(t)-\omega D) \theta(t) .
$$

Il existe un unique nombre réel $\mu(\omega)$ tel que $e^{\mu(\omega) \mathcal{T}}$ soit le multiplicateur de Floquet dominant du système $\frac{d X}{d t}=(Q(t)-\omega D) X$, qui est $\mathcal{T}$-périodique, avec une matrice à coefficients hors diagonale positifs ou nuls et irréductible sur un intervalle de $t$. On peut montrer qu'il existe un unique $\omega>0$ tel que $\mu(\omega)=0$ en utilisant, comme dans la section 9, la convexité de $\mu(\omega), \mu(0)=0$, le fait que $\mu^{\prime}(0)=-\frac{1}{\mathcal{T}} \int_{0}^{\mathcal{T}} \mathbf{1} D u(t) d t<0$ si $R_{0}<1$, et le fait que $\mu(\omega) \rightarrow+\infty$ lorsque $a_{J}<b$. 
Noter que la convexité de $\mu(\omega)$ se démontre en généralisant simplement l'argument de Cohen (1981), qui se base sur la log-convexité du rayon spectral des matrices à coefficients dans l'ensemble $\mathfrak{S}$ de fonctions nuls ou log-convexes en $\omega$, et sur le fait que $\mathfrak{S}$ est stable par addition, multiplication, et passage à la limite (Kingman, 1961). En effet, la fonction périodique continue par morceaux $Q(t)$ peut être approchée par une fonction en escalier, disons par une matrice $Q_{k}$ sur l'intervalle $\left(\tau_{k}, \tau_{k+1}\right)$ pour $k=0, \ldots, K-1$ avec $\tau_{0}=0$ et $\tau_{K}=\mathcal{T}$. Si $Q(t)$ était cette fonction en escalier, $e^{\mu(\omega) \mathcal{T}}$ serait égal au rayon spectral du produit de matrices $e^{\left(Q_{K-1}-\omega D\right)\left(\tau_{K}-\tau_{K-1}\right)} \times \cdots \times e^{\left(Q_{0}-\omega D\right)\left(\tau_{1}-\tau_{0}\right)}$. D'après Cohen (1981), chacune de ces matrices a ses coefficients dans $\mathfrak{S}$. Donc la matrice produit est aussi à coefficients dans $\mathfrak{S}$ et son rayon spectral est log-convexe en $\omega$.

Finalement, comme

$$
\lambda=-b \frac{\int_{0}^{\mathcal{T}} \sum_{j=1}^{J} \pi_{1, j}(t) d t}{\int_{0}^{\mathcal{T}} \sum_{j=1}^{J} \sum_{n=1}^{N} \pi_{n, j}(t) d t},
$$

on conclut comme avant que $-1 / \lambda$ croît sans doute avec $N$ comme $N^{\omega}$.

Remerciements Cet article a été stimulé par une réunion à l'Instituto de Ciencias Matemáticas à Madrid en octobre 2014, par des cours à l'université de Tlemcen en mai 2015 et par un séminaire à l'Universidade Nova de Lisboa en juin 2015 .

\section{Références}

Artalejo JR, Economou A, Lopez-Herreo MJ (2013) Stochastic epidemic models with random environment : quasi-stationarity, extinction and final size. J Math Biol 67 : 799-831

Bacaër N, Ed-Darraz A (2014) On linear birth-and-death processes in a random environment. J Math Biol 69 : 73-90

Bacaër N (2015) On the stochastic SIS epidemic model in a periodic environment. J Math Biol 71 : 491-511

Bender CM, Orszag SA (1978) Advanced mathematical methods for scientists and engineers. McGraw Hill, New York

Berman A, Plemmons RJ (1979) Nonnegative matrices in the mathematical sciences. Academic Press, New York

Cogburn R, Torrez WC (1981) Birth and death processes with random environments in continuous time. J Appl Probab 18 : 19-30

Cohen JE (1981) Convexity of the dominant eigenvalue of an essentially nonnegative matrix. Proc Amer Math Soc 81 : 657-658 
Doering CR, Sargsyan KV, Sander LM (2005) Extinction times for birth-death processes : exact results, continuum asymptotics, and the failure of the FokkerPlanck approximation. Multiscale Model Simul 3 : 283-299

Gaveau B, Moreau M, Toth J (1996) Decay of the metastable state in a chemical system : different predictions between discrete and continuous models. Lett Math Phys $37: 285-292$

Kamenev A, Meerson B, Shklovskii B (2008) How colored environmental noise affects population extinction. Phys Rev Lett $101: 268103$

Kato T (1982) Superconvexity of the spectral radius, and convexity of the spectral bound and the type. Math Z $180: 265-273$

Kingman JFC (1961) A convexity property of positive matrices. Quart J Math Oxford Ser (2) $12: 283-284$

Lande R (1993) Risks of population extinction from demographic and environmental stochasticity and random catastrophes. Am Nat 142 : 911-927

Leigh EG (1981) The average lifetime of a population in a varying environment. J Theor Biol $90: 213-239$

Ludwig D (1976) A singular perturbation problem in the theory of population extinction. SIAM-AMS Proceedings $10: 87-104$

Maroni P (1997) Fonctions hypergéométriques, fonctions de Bessel. Éditions Techniques de l'Ingénieur, Paris

Meerson B, Sasorov PV (2008) Noise-driven unlimited population growth. Phys Rev E 78 : 060103

Nåsell I (2011) Extinction and quasi-stationarity in the stochastic logistic SIS model. Springer, Berlin

Slatkin M (1978) The dynamics of a population in a Markovian environment. Ecology $59: 249-256$

Sawyer S, Slatkin M (1981) Density independent fluctuations of population size. Theor Popul Biol 19 : 37-57

Smith HL (1995) Monotone dynamical systems. American Mathematical Society, Providence RI 